\title{
DIVERGENCE BOUNDARY CONDITIONS FOR VECTOR HELMHOLTZ EQUATIONS WITH DIVERGENCE CONSTRAINTS
}

\author{
URVE KANGRO $^{1}$ AND RoY NiCOLAIDES ${ }^{2}$
}

\begin{abstract}
The idea of replacing a divergence constraint by a divergence boundary condition is investigated. The connections between the formulations are considered in detail. It is shown that the most common methods of using divergence boundary conditions do not always work properly. Necessary and sufficient conditions for the equivalence of the formulations are given.
\end{abstract}

AMS Subject Classification. 35L05, 65N12, 65M17.

Received: January 1998.

\section{INTRODUCTION}

A well known problem in computational electromagnetics is the appearance of "spurious modes" arising from incorrectly imposing divergence constraints. To avoid imposing these constraints directly, which is difficult, it has been suggested that they need to be enforced only on the boundary of the domain $[5,7]$. This has some significant advantages. For instance, the vector Helmholtz equation with a divergence constraint on the field can then be solved using standard finite element spaces instead of more complex spaces of edge elements. Furthermore, as we will show below, the divergence boundary condition can be treated as a natural boundary condition.

It is essential to know when the interior and boundary formulations of divergence constraints give the same result and that is the main aim of this work. We shall show that the formulations are equivalent if and only if the scalar Poisson equation with any smooth right hand side and Dirichlet boundary conditions has a solution in $H^{2}$. When this is not true, a simple minded application of divergence boundary conditions as in [5] and [7] will normally give incorrect solutions with a nonzero divergence.

Our results for systems with the interior divergence constraint are obtained by formulating the problem in the space $V$ defined in the next section. This approach avoids enforcing the divergence constraint explicitly it is automatically satisfied. We provide a proof of this fact for interior problems; for exterior domains see [4]. Unfortunately, $H^{1}$ is not dense in $V$ in general. To use standard $H^{1}$ finite elements we impose the divergence condition on the boundary and formulate the problem in the space $H_{0 t}^{1} \subset H^{1}$ defined below. We discuss the relationship between the $V$ and $H_{0 t}^{1}$ formulations in some detail.

Keywords and phrases. Maxwell's equations, divergence boundary conditions, Helmholtz equation, divergence constraints.

1 Department of Mathematics, Tartu University, Tartu, Estonia. e-mail: ukangro@ut.ee

2 Department of Mathematical Sciences, Carnegie Mellon University, Pittsburgh, PA 15213, USA. e-mail: rn0m@andrew.cmu.edu The work of this author was supported by the United States Air Force Office of Scientific Research under grants F-49620-94-10311 and F-49620-97-1-0204. 
To avoid relatively unimportant technical issues we will give proofs of these results for the vector Poisson equation subject to tangential boundary conditions and a divergence constraint. In the end we will show how they may be extended to other problems including the vector Helmholtz case of electromagnetics.

The next section gives the strong forms of the governing equations and the reasoning behind the use of divergence boundary conditions. It also contains a formula for the boundary divergence, which is used to reformulate the divergence boundary condition as a natural boundary condition in $H^{1}$ setting. Section 3 sets up a weak form for the equation with the interior divergence constraint and proves the coercivity for the weak form, which follows from a compact embedding result for vector fields. Sections 4 and 5 prove coercivity for the weak form which uses divergence boundary conditions. This time, coercivity follows from a close and somewhat surprising connection with the previous weak form. This result is based on a trace theorem proved in Section 5 .

The coercivity results of Sections 3-5 are used in Section 6 to prove equivalence of the strong and weak formulations (Theorem 2). We also discuss in what sense the boundary divergence condition is satisfied and prove a formula for calculating the divergence on the boundary (Lemma 10 in Sect. 6). Section 7 contains a discussion of when one can say a priori that the interior and boundary divergence formulations are equivalent, and when they are not equivalent (i.e. may have different solutions for the same data). It also contains an example showing nonequivalence of the two formulations for nonconvex polyhedra. In Section 8 we give the modifications to include the Helmholtz equation and briefly mention some other extensions. Section 9 mentions some implications for numerical approximations of the problem.

\section{Formulation of the problems}

Assume that $\Omega$ is an open bounded subset of $\mathbf{R}^{N}, N=2$ or 3 , with a connected, Lipchitz boundary $\Gamma$. We will consider the relationship between the problems

$$
\begin{cases}-\Delta \mathbf{u}=\mathbf{f} & \text { in } \Omega \\ \operatorname{div} \mathbf{u}=0 & \text { in } \Omega \\ \mathbf{u} \times \mathbf{n}=0 & \text { on } \Gamma\end{cases}
$$

and

$$
\begin{cases}-\triangle \mathbf{u}=\mathbf{f} & \text { in } \Omega \\ \operatorname{div} \mathbf{u}=0 & \text { on } \Gamma \\ \mathbf{u} \times \mathbf{n}=0 & \text { on } \Gamma\end{cases}
$$

We assume that $\mathbf{f} \in L^{2}(\Omega)^{N}$ and the compatibility condition $\operatorname{div} \mathbf{f}=0$ is satisfied. Superficially it seems that the two problems are equivalent. Indeed, if $\mathbf{u}$ is a solution of (1), it is clearly a solution of (2). Conversely, if $\mathbf{u}$ is a solution of (2), then taking the divergence of the first equation of (2) we get that $\triangle($ div $\mathbf{u})=0$, and since $\operatorname{div} \mathbf{u}=0$ on $\Gamma$, one might argue that $\operatorname{div} \mathbf{u}=0$ in $\Omega$, so $\mathbf{u}$ is a solution of (1). This is basically the reasoning in [7]. But it might not always be true. The difficulty is caused by insufficient regularity of the solution. Laplace's equation with Dirichlet boundary conditions has a unique solution in $H^{1}(\Omega)$, but in general we cannot assume that $\operatorname{div} \mathbf{u} \in H^{1}(\Omega)$. It may be only in $L^{2}(\Omega)$ and the homogeneous equation for div $\mathbf{u}$ may have a nonzero solution in $L^{2}(\Omega)$ (this happens, for example, in the case of domains with reentrant corners).

In order to discuss the the relationship between (1) and (2) we first have to make explicit in what function spaces we look for the solutions. It turns out that the natural space for (1) is

$$
V=\left\{\mathbf{u} \in L^{2}(\Omega)^{N}\left|\operatorname{div} \mathbf{u} \in L^{2}(\Omega), \operatorname{curl} \mathbf{u} \in L^{2}(\Omega)^{N^{\prime}}, \mathbf{u} \times \mathbf{n}\right|_{\Gamma}=0\right\},
$$

where $N^{\prime}=3$ if $N=3$ and $N^{\prime}=1$ in $N=2$. The standard scalar product in $V$ is

$$
(\mathbf{u}, \mathbf{v})_{V}=\int_{\Omega} \mathbf{u} \cdot \mathbf{v}+\operatorname{curl} \mathbf{u} \cdot \operatorname{curl} \mathbf{v}+\operatorname{div} \mathbf{u} \operatorname{div} \mathbf{v}
$$


On the other hand, the natural space for (2) is

$$
H_{0 t}^{1}(\Omega)=\left\{\mathbf{u} \in H^{1}(\Omega)^{N}|\mathbf{u} \times \mathbf{n}|_{\Gamma}=0\right\}
$$

with the scalar product

$$
(\mathbf{u}, \mathbf{v})_{1}=\int_{\Omega} \mathbf{u} \cdot \mathbf{v}+\nabla \mathbf{u} \cdot \nabla \mathbf{v}
$$

We will show that while problem (1) has a unique solution in $V$, it may not be solvable in $H_{0 t}^{1}(\Omega)$, and while problem (2) has a unique solution in $H_{0 t}^{1}(\Omega)$, the solution may not be unique in $V$. We will also discuss in what sense the divergence boundary condition in problem (2) is satisfied.

It is shown in Section 6 (under the additional assumptions that $\Gamma$ is piecewise $C^{1,1}$ and the jump condition given in (6) is satisfied) that problem $(2)$ (in $H_{0 t}^{1}(\Omega)$ ) is equivalent to: find $\mathbf{u} \in H_{0 t}^{1}(\Omega)$ such that

$$
\begin{cases}-\triangle \mathbf{u}=\mathbf{f} & \text { in } \Omega \\ \frac{\partial \mathbf{u}}{\partial \mathbf{n}} \cdot \mathbf{n}+\kappa \mathbf{u} \cdot \mathbf{n}=0 & \text { on } \Gamma \\ \mathbf{u} \times \mathbf{n}=0 & \text { on } \Gamma\end{cases}
$$

where $\kappa$ is the curvature of the boundary $(\kappa=\operatorname{div} \mathbf{n})$, which is defined almost everywhere on $\Gamma$. This follows from the result (see Lemma 10 for the precise formulation)

$$
\left.\operatorname{div} \mathbf{u}\right|_{\Gamma}=\left.\frac{\partial \mathbf{u}}{\partial \mathbf{n}} \cdot \mathbf{n}\right|_{\Gamma}+\left.\kappa \mathbf{u} \cdot \mathbf{n}\right|_{\Gamma}
$$

It is convenient to begin with the weak formulations of problems (1) and (3). The next three sections deal with the coercivity of the corresponding weak forms. Following this we show the equivalence of the strong and weak problems and then examine the relationship of problems (1) and (3), and show the equivalence of problems (2) and (3).

\section{WEAK FORMULATiON IN V}

Let $\Omega$ and $\mathbf{f}$ be as above. To derive the weak formulation of (1) in $V$ we substitute curl curl $\mathbf{u}$ for $-\triangle \mathbf{u}$ in (1), multiply the first equation by $\mathbf{v}$ (belonging to $V$ ), the second by div $\mathbf{v}$ and integrate by parts using the boundary condition. For $\mathbf{u}, \mathbf{v} \in V$ define

$$
a(\mathbf{u}, \mathbf{v})=\int_{\Omega} \operatorname{curl} \mathbf{u} \cdot \operatorname{curl} \mathbf{v}+\operatorname{div} \mathbf{u} \operatorname{div} \mathbf{v} .
$$

Then the statement of the weak problem is: find $\mathbf{u} \in V$ such that

$$
a(\mathbf{u}, \mathbf{v})=\int_{\Omega} \mathbf{f} \cdot \mathbf{v} \quad \forall \mathbf{v} \in V .
$$

The only hypothesis of the Lax-Milgram lemma which is nontrivial to check is the coercivity of $a$ on $V$. It follows from the next compact embedding theorem. The embedding is actually a corollary of the regularity result in [1], where it is proved that $V \subset H^{1 / 2}(\Omega)$. We have provided a new and concise proof.

Theorem 1. $V$ is compactly embedded in $L^{2}(\Omega)^{N}$. 
The proof makes use of Murat's div-curl lemma (see [8]).

Lemma 1. (div-curl lemma). Let $U$ be an open subset of $\mathbf{R}^{N}$. Let $\mathbf{v}_{n}$ and $\mathbf{w}_{n}$ be weakly convergent sequences in $L^{2}(U)^{N}$ with the limits $\mathbf{v}$ and $\mathbf{w}$ correspondingly. Assume that $\left\{\operatorname{div} \mathbf{v}_{n}\right\}_{n=1}^{\infty}$ and $\left\{\mathbf{c u r l} \mathbf{w}_{n}\right\}_{n=1}^{\infty}$ lie in compact subsets of $H^{-1}(U)$ and $H^{-1}(U)^{N^{\prime}}$ respectively. Then for every $\phi \in \mathcal{D}(U)$,

$$
\int_{U} \phi \mathbf{v}_{n} \cdot \mathbf{w}_{n} \rightarrow \int_{U} \phi \mathbf{v} \cdot \mathbf{w} \text { as } n \rightarrow \infty
$$

Proof of Theorem 1. Let $\mathbf{u}_{n}$ be a weakly convergent sequence in $V$ with the limit $\mathbf{u}$. We want to show that $\mathbf{u}_{n}$ converges strongly in $L^{2}(\Omega)$. The idea is to extend $\mathbf{u}_{n}$ outside $\Omega$ in two ways: $\mathbf{v}_{n}$ and $\mathbf{w}_{n}$ will be the extensions with "good" divergence and curl respectively. We construct the extensions so that $\operatorname{supp} \mathbf{v}_{n} \cap \operatorname{supp} \mathbf{w}_{n} \subset \bar{\Omega}$ and then use div-curl lemma for these sequences.

Let $U$ be an open ball containing $\Omega$. We start with the construction of $\mathbf{v}_{n}$ (the extension with $\operatorname{div} \mathbf{v}_{n}$ in a compact set of $\left.H^{-1}(U)\right)$. For each $n \in \mathbf{N}$ we define $g_{n} \in H^{1}(U \backslash \Omega)$ by

$$
\begin{cases}\triangle g_{n}=0 & \text { in } U \backslash \Omega \\ \frac{\partial g_{n}}{\partial \mathbf{n}}=\mathbf{u}_{n} \cdot \mathbf{n} & \text { on } \partial \Omega \\ g_{n}=0 & \text { on } \partial U\end{cases}
$$

Now define $\mathbf{v}_{n}$ by

$$
\mathbf{v}_{n}= \begin{cases}\mathbf{u}_{n} & \text { in } \Omega \\ \nabla g_{\text {in }} & \text { in } U \backslash \Omega\end{cases}
$$

and $\mathbf{w}_{n}$ by

$$
\mathbf{w}_{n}= \begin{cases}\mathbf{u}_{n} & \text { in } \Omega \\ 0 & \text { in } U \backslash \Omega\end{cases}
$$

Then

$$
\operatorname{div} \mathbf{v}_{n}= \begin{cases}\operatorname{div} \mathbf{u}_{n} & \text { in } \Omega, \\ 0 & \text { in } U \backslash \Omega\end{cases}
$$

and

$$
\operatorname{curl}_{n}= \begin{cases}\operatorname{curl} \mathbf{u}_{n} & \text { in } \Omega, \\ 0 & \text { in } U \backslash \Omega\end{cases}
$$

so $\left\{\operatorname{div} \mathbf{v}_{n}\right\}_{n=1}^{\infty}$ and $\left\{\operatorname{curl}_{\mathbf{l}} \mathbf{w}_{n}\right\}_{n=1}^{\infty}$ are bounded subsets of $L^{2}(U)$ and $L^{2}(\Omega)^{N^{\prime}}$, respectively (hence lie in compact sets of $H^{-1}(U)$ and $\left.H^{-1}(\Omega)^{N^{\prime}}\right)$. Moreover, $\mathbf{v}_{n} \rightarrow \mathbf{v}$ in $L^{2}(U)^{N}$ and $\mathbf{w}_{n} \rightarrow \mathbf{w}$ in $L^{2}(U)^{N}$, where $\mathbf{v}$ and $\mathbf{w}$ are similar extensions of $\mathbf{u}$.

Now choose $\phi \in \mathcal{D}(U)$ such that $\phi \equiv 1$ in $\Omega$. Using the div-curl lemma we get (note that $\mathbf{v}_{n} \cdot \mathbf{w}_{n}=0$ in $U \backslash \Omega)$

$$
\int_{\Omega} \mathbf{u}_{n}^{2}=\int_{U} \phi \mathbf{v}_{n} \cdot \mathbf{w}_{n} \rightarrow \int_{U} \phi \mathbf{v} \cdot \mathbf{w}=\int_{\Omega} \mathbf{u}^{2}
$$

This together with $\mathbf{u}_{n} \rightarrow \mathbf{u}$ in $L^{2}(\Omega)$ shows that $\mathbf{u}_{n} \rightarrow \mathbf{u}$ in $L^{2}(\Omega)$. 
We will use Peetre's lemma (see [2], for example) in the following form

Lemma 2. Let $E, E_{1}$ and $E_{2}$ be Banach spaces. Let $A_{1}$ and $A_{2}$ be continuous linear operators from $E$ to $E_{1}$ and from $E$ to $E_{2}$ respectively. Assume that there exists $C>0$ such that

$$
\|u\|_{E} \leq C\left(\left\|A_{1} u\right\|_{E_{1}}+\left\|A_{2} u\right\|_{E_{2}}\right) \quad \forall u \in E .
$$

Assume also that $\operatorname{Ker} A_{1}=\{0\}$ and that $A_{2}$ is compact. Then there is $C_{1}>0$ such that

$$
\|u\|_{E} \leq C_{1}\left\|A_{1} u\right\|_{E_{1}} \quad \forall u \in E .
$$

The coercivity result is then

Lemma 3. There is $C>0$, independent of $\mathbf{v}$, such that

$$
a(\mathbf{v}, \mathbf{v}) \geq C\|\mathbf{v}\|_{V}^{2} \quad \forall \mathbf{v} \in V .
$$

Proof. We use Lemma 2 with $E=V, E_{1}=L^{2}(\Omega) \times L^{2}(\Omega)^{N^{\prime}}, E_{2}=L^{2}(\Omega)^{N}$ and $A_{1} \mathbf{u}=(\operatorname{div} \mathbf{u}$, curl u), $A_{2} \mathbf{u}=\mathbf{u}$. By Lemma 1 the operator $A_{2}$ is compact. We must show that Ker $A_{1}=\{0\}$. But

$$
\mathbf{u} \in \operatorname{Ker} A_{1} \Leftrightarrow \operatorname{div} \mathbf{u}=0 \text { and } \operatorname{curl} \mathbf{u}=0 \text { in } \Omega \text {, and } \mathbf{u} \times \mathbf{n}=0 \text { on } \Gamma .
$$

This implies that $\mathbf{u}=0$ (by using the gradient potential, for example). So by Lemma 2 the result follows.

The Lax-Milgram lemma now implies that (5) has a unique, stable solution in $V$.

\section{WEAK FORMULATiON IN $\mathrm{H}_{0 t}^{1}(\Omega)$}

For deriving the weak form of (3) we shall make additional assumptions about the smoothness of the boundary. In the following $\Omega$ is a bounded subset of $\mathbf{R}^{N}, N=2$ or 3 , with a connected, Lipchitz and piecewise $C^{1,1}$ boundary $\Gamma$, i.e. $\Gamma=\cup_{j=1}^{n} \Gamma_{j}$ with $\stackrel{\circ}{\Gamma}_{j} \cap \stackrel{\circ}{\Gamma}_{i}=\emptyset$ for $i \neq j$, and $\Gamma_{j}, j=1, \ldots, n$ are of class $C^{1,1}$. Let $\gamma$ denote the set of "edges and corners", i.e.

$$
\gamma=\bigcup_{\substack{i, j=1 \\ i \neq j}}^{n}\left(\bar{\Gamma}_{i} \cap \bar{\Gamma}_{j}\right) .
$$

We assume that the jump of the normal on $\gamma$ is bounded below, i.e. there is $\delta>0$ such that for all $\mathbf{x} \in \gamma$ with $\mathbf{x} \in \bar{\Gamma}_{i} \cap \bar{\Gamma}_{j}, i \neq j$ we have

$$
\mathbf{n}^{i}(\mathbf{x}) \cdot \mathbf{n}^{j}(\mathbf{x}) \leq 1-\delta
$$

where $\mathbf{n}^{i}(\mathbf{x})$ and $\mathbf{n}^{j}(\mathbf{x})$ are the limits of the unit outer normals when approaching $\mathbf{x}$ from $\Gamma_{i}$ and $\Gamma_{j}$, correspondingly.

This condition is satisfied for all polygons and polyhedra. Also included are polygons and polyhedra with curved sides. It excludes three-dimensional bodies which have points like the tip of a cone, and bodies with edges which "flatten out", i.e. the angle between the faces gets arbitrarily close to $\pi$. This condition is needed for proving some results about trace operators in the next section, and implicitly in the proof of Lemma 4, which gives the relation between the two weak forms.

To obtain a suitable weak form for (3) we proceed formally, multiplying the equation by a test function $\mathbf{v} \in H_{0 t}^{1}(\Omega)$ and integrating by parts as usual. We get

$$
\int_{\Omega} \nabla \mathbf{u} \cdot \nabla \mathbf{v}-\int_{\Gamma} \frac{\partial \mathbf{u}}{\partial \mathbf{n}} \cdot \mathbf{v}=\int_{\Omega} \mathbf{f} \cdot \mathbf{v} .
$$


Rewriting the boundary term as a sum over the smooth boundary pieces and using the boundary conditions on $\mathbf{u}$ and $\mathbf{v}$ gives the weak problem: find $\mathbf{u} \in H_{0 t}^{1}(\Omega)$ such that

$$
\int_{\Omega} \nabla \mathbf{u} \cdot \nabla \mathbf{v}+\sum_{j=1}^{n} \int_{\Gamma_{j}} \kappa \mathbf{u} \cdot \mathbf{v}=\int_{\Omega} \mathbf{f} \cdot \mathbf{v} \quad \forall \mathbf{v} \in H_{0 t}^{1}(\Omega) .
$$

Since $\kappa \in L^{\infty}\left(\Gamma_{j}\right)$, by the usual trace theorem the boundary term is well defined. Note that, in this framework, the divergence boundary condition (or the equivalent one in problem (3)) is natural.

Proving coercivity of the weak form (7) is a nontrivial matter because the curvature can be of either sign. The result will follow from the next lemma whose proof is deferred to the following section.

Lemma 4. Assume that $\Omega$ satisfies the assumptions made in the beginning of the section. For $\mathbf{u}, \mathbf{v} \in H_{0 t}^{1}(\Omega)$ we have

$$
a(\mathbf{u}, \mathbf{v})=\int_{\Omega} \nabla \mathbf{u} \cdot \nabla \mathbf{v}+\sum_{j=1}^{n} \int_{\Gamma_{j}} \kappa \mathbf{u} \cdot \mathbf{v}
$$

where $\Gamma_{j}, j=1, \ldots, n$ are the smooth $\left(C^{1,1}\right)$ pieces of $\Gamma$, and a is the same as in (4).

This means that the weak problem $(7)$ is in fact equivalent to: Find $\mathbf{u} \in H_{0 t}^{1}(\Omega)$ such that

$$
a(\mathbf{u}, \mathbf{v})=\int_{\Omega} \mathbf{f} \cdot \mathbf{v} \forall \mathbf{v} \in H_{0 t}^{1}(\Omega)
$$

in which the equation is the same as in (5) but the spaces are different.

In fact, one can get (9) from problem (1), but they are not equivalent: in general we cannot assume that the solution of $(1)$ belongs to $H_{0 t}^{1}(\Omega)$, and we cannot prove later that the solution of $(9)$ satisfies div $\mathbf{u}=0$. We can also formally get (9) from (2) by substituting $-\triangle \mathbf{u}$ by curl curl $\mathbf{u}-\nabla \operatorname{div} \mathbf{u}$, multiplying by $\mathbf{v}$ and integrating by parts using the boundary conditions, but this is not easy to justify rigorously (for the solution of (2), curl curl $\mathbf{u}$ and $\nabla \operatorname{div} \mathbf{u}$ may not be in $L^{2}$ ).

The coercivity result for $(9)$ is:

Lemma 5. There is $C>0$ such that

$$
a(\mathbf{u}, \mathbf{v}) \geq C\|\mathbf{u}\|_{1} \quad \forall \mathbf{u} \in H_{0 t}^{1}(\Omega)
$$

Proof. We use Lemma 2 with $E=H_{0 t}^{1}(\Omega), E_{1}=L^{2}(\Omega) \times L^{2}(\Omega)^{N^{\prime}}, E_{2}=L^{2}(\Omega)^{N} \times L^{2}(\Gamma)^{N}, A_{1} \mathbf{u}=(\operatorname{div} \mathbf{u}, \mathbf{c u r l} \mathbf{u})$ and $A_{2} \mathbf{u}=\left(\mathbf{u},\left.\mathbf{u}\right|_{\Gamma}\right)$. The operator $A_{2}: E \rightarrow E_{2}$ is compact because it is bounded as an operator from $E$ to $H^{1}(\Omega) \times H^{1 / 2}(\Gamma)^{N}$ and the latter is compactly embedded into $E_{2}$. We already showed (in the proof of Lemma 3 ) that $\operatorname{Ker} A_{1}=\{0\}$. The result follows.

Applying the Lax-Milgram lemma to the weak form (9) (or, equivalently, to (9)) and using the coercivity result above shows the existence of a unique stable solution to (9).

Note that to prove the coercivity of $a$ in $H_{0 t}^{1}(\Omega)$ we do not need the additional smoothness assumptions made in the beginning of this section: they are needed to make sense of the term containing the curvature in (7) and to show later that the divergence boundary condition is satisfied for the solution of problem (9). They are not needed for uniqueness of solution of (9).

\section{Proofs For PREvious SECTION}

This section will present the proof of Lemma 4. We will assume throughout the section that the assumptions made in the beginning of the previous section are satisfied. 
First we need some preliminary results. We begin by recalling some facts about the trace spaces. First, for Lipchitz $\Gamma \subset \mathbf{R}^{N}$, the following is an equivalent norm on $H^{1 / 2}(\Gamma)$ (see e.g. [3]):

$$
\|\phi\|_{H^{1 / 2}(\Gamma)}^{2}=\|\phi\|_{L^{2}(\Gamma)}^{2}+\int_{\Gamma} \int_{\Gamma} \frac{|\phi(\mathbf{x})-\phi(\mathbf{y})|^{2}}{|\mathbf{x}-\mathbf{y}|^{N}} d S_{\mathbf{x}} d S_{\mathbf{y}} .
$$

We will also need the spaces $H_{00}^{1 / 2}\left(\Gamma_{j}\right), j=1, \ldots, n$, which consist of all functions in $H^{1 / 2}\left(\Gamma_{j}\right)$ whose extension by zero to $\Gamma$ belongs to $H^{1 / 2}(\Gamma)$. The norm of a function in $H_{00}^{1 / 2}\left(\Gamma_{j}\right)$ is the norm of its extension by zero in $H^{1 / 2}(\Gamma)$, i.e.

$$
\begin{aligned}
\|\phi\|_{H_{00}^{1 / 2}\left(\Gamma_{j}\right)}^{2}= & \|\phi\|_{L^{2}\left(\Gamma_{j}\right)}^{2}+\int_{\Gamma_{j}} \int_{\Gamma_{j}} \frac{|\phi(\mathbf{x})-\phi(\mathbf{y})|^{2}}{|\mathbf{x}-\mathbf{y}|^{N}} d S_{\mathbf{x}} d S_{\mathbf{y}} \\
& +2 \int_{\Gamma_{\lceil} \Gamma_{j}} \int_{\Gamma_{j}} \frac{|\phi(\mathbf{x})|^{2}}{|\mathbf{x}-\mathbf{y}|^{N}} d S_{\mathbf{x}} d S_{\mathbf{y}} .
\end{aligned}
$$

We will use the same notation for a function in $H_{00}^{1 / 2}\left(\Gamma_{j}\right)$ and its extension by zero to $\Gamma$.

We will also need the following result:

Lemma 6. The trace operator $\left.\mathbf{v} \mapsto \mathbf{v} \cdot \mathbf{n}\right|_{\Gamma_{j}}$ is a continuous linear operator from $H_{0 t}^{1}(\Omega)$ onto $H_{00}^{1 / 2}\left(\Gamma_{j}\right)$.

Proof. We use the fact that the usual trace operator $\left.\mathbf{v} \mapsto \mathbf{v}\right|_{\Gamma}$ is a continuous linear operator from $H^{1}(\Omega)^{N}$ onto $H^{1 / 2}(\Gamma)^{N}$. First we will show that if $\mathbf{v} \times \mathbf{n}=0$ and the jump condition (6) is satisfied, then $\|\mathbf{v} \cdot \mathbf{n}\|_{H_{00}^{1 / 2}\left(\Gamma_{j}\right)} \leq$ $C\|\mathbf{v}\|_{H^{1 / 2}(\Gamma)}$. Let $\phi=\mathbf{v} \cdot \mathbf{n}$ on $\Gamma_{j}$ and $\phi \equiv 0$ on $\Gamma \backslash \Gamma_{j}$. Clearly $\|\phi\|_{H^{1 / 2}\left(\Gamma_{j}\right)} \leq\|\mathbf{v}\|_{H^{1 / 2}\left(\Gamma_{j}\right)}$, so we have to show only that

$$
\int_{\Gamma \backslash \Gamma_{j}} \int_{\Gamma_{j}} \frac{|\phi(\mathbf{x})|^{2}}{|\mathbf{x}-\mathbf{y}|^{N}} d S_{\mathbf{x}} d S_{\mathbf{y}} \leq C\|\mathbf{v}\|_{H^{1 / 2}\left(\Gamma_{j}\right)}^{2}
$$

Because of the jump condition we can find $\varepsilon>0$ such that

$$
\mathbf{x} \in \Gamma_{j}, \mathbf{y} \in \Gamma \backslash \Gamma_{j},|\mathbf{x}-\mathbf{y}|<\varepsilon \Rightarrow \mathbf{n}(\mathbf{x}) \cdot \mathbf{n}(\mathbf{y}) \leq 1-\frac{\delta}{2} .
$$

For $\mathbf{x} \in \Gamma_{j}, \mathbf{y} \in \Gamma \backslash \Gamma_{j}$ such that $|\mathbf{x}-\mathbf{y}|<\varepsilon$ we have

$$
\begin{aligned}
|\mathbf{v}(\mathbf{x})-\mathbf{v}(\mathbf{y})|^{2} & =|\mathbf{v}(\mathbf{x})|^{2}+|\mathbf{v}(\mathbf{y})|^{2}-2(\mathbf{v}(\mathbf{x}) \cdot \mathbf{n}(\mathbf{x}))(\mathbf{v}(\mathbf{y}) \cdot \mathbf{n}(\mathbf{y}))(\mathbf{n}(\mathbf{x}) \cdot \mathbf{n}(\mathbf{y})) \\
& \geq|\phi(\mathbf{x})|^{2}+|\mathbf{v}(\mathbf{y})|^{2}-2|\phi(\mathbf{x})||\mathbf{v}(\mathbf{y})|\left(1-\frac{\delta}{2}\right) \\
& \geq|\phi(\mathbf{x})|^{2}\left(1-\left(1-\frac{\delta}{2}\right)^{2}\right)=|\phi(\mathbf{x})|^{2}\left(\delta-\frac{\delta^{2}}{4}\right)
\end{aligned}
$$

Since $\delta-\frac{\delta^{2}}{4}>0$ (note that $0<\delta<2$, otherwise the jump condition could not be satisfied), we have

$$
\begin{aligned}
\int_{\Gamma \backslash \Gamma_{j}} \int_{\Gamma_{j}} \frac{|\phi(\mathbf{x})|^{2}}{|\mathbf{x}-\mathbf{y}|^{N}} d S_{\mathbf{x}} d S_{\mathbf{y}} & =\int_{\Gamma \backslash \Gamma_{j}} \int_{\Gamma_{j} \backslash B_{\varepsilon}(\mathbf{y})} \frac{|\phi(\mathbf{x})|^{2}}{|\mathbf{x}-\mathbf{y}|^{N}} d S_{\mathbf{x}} d S_{\mathbf{y}}+\int_{\Gamma \backslash \Gamma_{j}} \int_{\Gamma_{j} \cap B_{\varepsilon}(\mathbf{y})} \frac{|\phi(\mathbf{x})|^{2}}{|\mathbf{x}-\mathbf{y}|^{N}} d S_{\mathbf{x}} d S_{\mathbf{y}} \\
& \leq \frac{1}{\varepsilon^{N}} \int_{\Gamma \backslash \Gamma_{j}} \int_{\Gamma_{j}}|\phi(\mathbf{x})|^{2} d S_{\mathbf{x}} d S_{\mathbf{y}}+\frac{1}{\delta-\frac{\delta^{2}}{4}} \int_{\Gamma \backslash \Gamma_{j}} \int_{\Gamma_{j}} \frac{|\mathbf{v}(\mathbf{x})-\mathbf{v}(\mathbf{y})|^{2}}{|\mathbf{x}-\mathbf{y}|^{N}} d S_{\mathbf{x}} d S_{\mathbf{y}} \\
& \leq C\|\mathbf{v}\|_{H^{1 / 2}(\Gamma)}^{2} .
\end{aligned}
$$


Consequently, the operator $\left.\mathbf{v} \mapsto \mathbf{v} \cdot \mathbf{n}\right|_{\Gamma_{j}}$ is a continuous linear operator from $H_{0 t}^{1}(\Omega)$ to $H_{00}^{1 / 2}\left(\Gamma_{j}\right)$.

Let $\phi \in H_{00}^{1 / 2}\left(\Gamma_{j}\right)$ be given. Extend it by 0 to $\Gamma$. Then $\phi \mathbf{n} \in H^{1 / 2}(\Gamma)$ and we can find $\mathbf{v} \in H^{1}(\Omega)^{N}$ such that $\left.\mathbf{v}\right|_{\Gamma}=\phi \mathbf{n}$ and $\|\mathbf{v}\|_{1} \leq C\|\phi \mathbf{n}\|_{H^{1 / 2}(\Gamma)} \leq C_{1}\|\phi\|_{H_{00}^{1 / 2}\left(\Gamma_{j}\right)}$. Clearly $\mathbf{v} \in H_{0 t}^{1}(\Omega)$, so the trace operator is onto.

The next lemma gives a formula for calculating the divergence on the boundary.

Lemma 7. For smooth $\mathbf{u}$ the restriction of $\operatorname{div} \mathbf{u}$ to $\Gamma_{j}$ satisfies

$$
\left.\operatorname{div} \mathbf{u}\right|_{\Gamma_{j}}=\left.\left(\operatorname{div}_{\Gamma} \mathbf{u}_{\tau}+\kappa \mathbf{u} \cdot \mathbf{n}+\frac{\partial \mathbf{u}}{\partial \mathbf{n}} \cdot \mathbf{n}\right)\right|_{\Gamma_{j}}
$$

where $\mathbf{u}_{\tau}$ is the tangential component of $\mathbf{u}$ and $\operatorname{div}_{\Gamma}$ is the divergence of a tangential vector field in the tangential coordinate system.

Proof. To calculate div $\mathbf{u}$ at some $\mathbf{x}_{0} \in \Gamma_{j}$ let us fix a coordinate system $\mathbf{i}, \mathbf{j}, \mathbf{k}$ (if $N=2$ then omit $\mathbf{j}$ ), where $\mathbf{i}$, $\mathbf{j}$ are tangent to $\Gamma$ at $\mathbf{x}_{0}$ and $\mathbf{k}$ is the normal at $\mathbf{x}_{0}$ (we can do this for every $\mathbf{x}_{0}$ in the interior of $\Gamma_{j}$ ). Denote $\mathbf{u}_{\tau}=\mathbf{u}-(\mathbf{u} \cdot \mathbf{n}) \mathbf{n}$. Then

$$
\begin{aligned}
\operatorname{div} \mathbf{u}\left(\mathbf{x}_{0}\right) & =\frac{\partial \mathbf{u}}{\partial \mathbf{i}}\left(\mathbf{x}_{0}\right) \cdot \mathbf{i}+\frac{\partial \mathbf{u}}{\partial \mathbf{j}}\left(\mathbf{x}_{0}\right) \cdot \mathbf{j}+\frac{\partial \mathbf{u}}{\partial \mathbf{k}}\left(\mathbf{x}_{0}\right) \cdot \mathbf{k} \\
& =\frac{\partial \mathbf{u}_{\tau}}{\partial \mathbf{i}}\left(\mathbf{x}_{0}\right) \cdot \mathbf{i}+\frac{\partial \mathbf{u}_{\tau}}{\partial \mathbf{j}}\left(\mathbf{x}_{0}\right) \cdot \mathbf{j}+\frac{\partial[(\mathbf{u} \cdot \mathbf{n}) \mathbf{n}]}{\partial \mathbf{i}}\left(\mathbf{x}_{0}\right) \cdot \mathbf{i}+\frac{\partial[(\mathbf{u} \cdot \mathbf{n}) \mathbf{n}]}{\partial \mathbf{j}}\left(\mathbf{x}_{0}\right) \cdot \mathbf{j}+\frac{\partial \mathbf{u}}{\partial \mathbf{n}}\left(\mathbf{x}_{0}\right) \cdot \mathbf{n}\left(\mathbf{x}_{0}\right) \\
& =\left.\left(\operatorname{div}_{\Gamma} \mathbf{u}_{\tau}+\kappa \mathbf{u} \cdot \mathbf{n}+\frac{\partial \mathbf{u}}{\partial \mathbf{n}} \cdot \mathbf{n}\right)\right|_{\mathbf{x}=\mathbf{x}_{0}},
\end{aligned}
$$

where

$$
\operatorname{div}_{\Gamma} \mathbf{u}_{\tau}\left(\mathbf{x}_{0}\right)=\frac{\partial \mathbf{u}_{\tau}}{\partial \mathbf{i}}\left(\mathbf{x}_{0}\right) \cdot \mathbf{i}+\frac{\partial \mathbf{u}_{\tau}}{\partial \mathbf{j}}\left(\mathbf{x}_{0}\right) \cdot \mathbf{j}
$$

Now we can prove Lemma 4 :

Proof of Lemma 4. For $\mathbf{v} \in H_{0 t}^{1}(\Omega)$ and smooth $\mathbf{u}$ (we do not require $\mathbf{u} \times \mathbf{n}=0$ yet, because it does not seem straightforward to show the density of smooth functions with zero tangential trace in $H_{0 t}^{1}(\Omega)$ ) we have

$$
\begin{aligned}
\int_{\Omega} \operatorname{curl} \mathbf{u} \cdot \operatorname{curl} \mathbf{v}+\operatorname{div} \mathbf{u} \operatorname{div} \mathbf{v} & =\int_{\Omega} \operatorname{curl} \mathbf{c u r l} \mathbf{u} \cdot \mathbf{v}-\nabla \operatorname{div} \mathbf{u} \cdot \mathbf{v}+\int_{\Gamma}(\operatorname{div} \mathbf{u}) \mathbf{v} \cdot \mathbf{n} \\
& =-\int_{\Omega} \triangle \mathbf{u} \cdot \mathbf{v}+\int_{\Gamma}(\operatorname{div} \mathbf{u}) \mathbf{v} \cdot \mathbf{n} \\
& =\int_{\Omega} \nabla \mathbf{u} \cdot \nabla \mathbf{v}+\int_{\Gamma}\left(\operatorname{div} \mathbf{u}-\frac{\partial \mathbf{u}}{\partial \mathbf{n}} \cdot \mathbf{n}\right) \mathbf{v} \cdot \mathbf{n}
\end{aligned}
$$

By Lemma 7

$$
\int_{\Omega} \operatorname{curl} \mathbf{u} \cdot \operatorname{curl} \mathbf{v}+\operatorname{div} \mathbf{u} \operatorname{div} \mathbf{v}=\int_{\Omega} \nabla \mathbf{u} \cdot \nabla \mathbf{v}+\sum_{j=1}^{n} \int_{\Gamma_{j}}\left(\operatorname{div}_{\Gamma} \mathbf{u}_{\tau}+\kappa \mathbf{u} \cdot \mathbf{n}\right) \mathbf{v} \cdot \mathbf{n} .
$$

For any $\phi \in H_{00}^{1 / 2}\left(\Gamma_{j}\right)$ (extended by zero to $\Gamma$ ) we can find $\mathbf{v} \in H_{0 t}^{1}(\Omega)$ such that $\left.\mathbf{v}\right|_{\Gamma}=\phi \mathbf{n}$ and $\|\mathbf{v}\|_{1} \leq$ $C\|\phi\|_{H_{00}^{1 / 2}\left(\Gamma_{j}\right)}$. From (10) we get

$$
\left|\int_{\Gamma_{j}}\left(\operatorname{div}_{\Gamma} \mathbf{u}_{\tau}+\kappa \mathbf{u} \cdot \mathbf{n}\right) \phi\right| \leq C^{\prime}\|\mathbf{v}\|_{1}\|\mathbf{u}\|_{1} \leq C^{\prime \prime}\|\phi\|_{H_{00}^{1 / 2}\left(\Gamma_{j}\right)}\|\mathbf{u}\|_{1}, \forall \phi \in H_{00}^{1 / 2}\left(\Gamma_{j}\right), \forall \mathbf{u} \text { smooth. }
$$


By density of smooth functions in $H^{1}(\Omega)$ and Hahn-Banach theorem we can continue the mapping $\mathbf{u} \mapsto\left(\operatorname{div}_{\Gamma} \mathbf{u}_{\tau}+\right.$ $\kappa \mathbf{u} \cdot \mathbf{n})\left.\right|_{\Gamma_{j}}$ uniquely to a continuous linear mapping from $H^{1}(\Omega)^{N}$ to $\left(H_{00}^{1 / 2}\left(\Gamma_{j}\right)\right)^{\prime}$. Since for any $\mathbf{v} \in H_{0 t}^{1}(\Omega)$, $\mathbf{v} \cdot \mathbf{n} \in H_{00}^{1 / 2}\left(\Gamma_{j}\right)$ (by Lemma 6), (10) holds for all $\mathbf{u} \in H^{1}(\Omega)^{N}$ and $\mathbf{v} \in H_{0 t}^{1}(\Omega)$ (the integrals over $\Gamma_{j}$ have a meaning as a duality pairing between $\left(H_{00}^{1 / 2}\left(\Gamma_{j}\right)\right)^{\prime}$ and $\left.H_{00}^{1 / 2}\left(\Gamma_{j}\right)\right)$.

To finish the proof we need to show only that if $\mathbf{u} \in H_{0 t}^{1}(\Omega)$, then $\operatorname{div}_{\Gamma} \mathbf{u}_{\tau}=0$ on $\Gamma_{j}$. For this first note that since $\left.\mathbf{u} \mapsto \kappa \mathbf{u} \cdot \mathbf{n}\right|_{\Gamma_{j}}$ is linear and continuous from $H^{1}(\Omega)^{N}$ to $L^{2}\left(\Gamma_{j}\right) \subset\left(H_{00}^{1 / 2}\left(\Gamma_{j}\right)\right)^{\prime}$, the operator $\left.\mathbf{u} \mapsto \operatorname{div}_{\Gamma} \mathbf{u}_{\tau}\right|_{\Gamma_{j}}$ is also continuous from $H^{1}(\Omega)^{N}$ to $\left(H_{00}^{1 / 2}\left(\Gamma_{j}\right)\right)^{\prime}$, and then use the fact that

$$
\left\langle\operatorname{div}_{\Gamma} \mathbf{u}_{\tau}, \phi\right\rangle_{\Gamma_{j}}=-\int_{\Gamma_{j}} \mathbf{u}_{\tau} \cdot \nabla_{\tau} \phi \forall \phi \in C_{c}^{1}\left(\Gamma_{j}\right)
$$

and the density of $C_{c}^{1}\left(\Gamma_{j}\right)$ in $H_{00}^{1 / 2}\left(\Gamma_{j}\right)$.

\section{EqUivalence OF THE STRONG AND WEAK FORMS}

In this section we will prove that (1) in $V$ is equivalent to the weak problem (5), and that $(3)$ in $H_{0 t}^{1}(\Omega)$ is equivalent to the weak problem (7). It follows that problem (1) is uniquely solvable in $V$ and problem (3) is uniquely solvable in $H_{0 t}^{1}(\Omega)$. We will also show that problems (2) and (3) are equivalent with the divergence boundary condition having a meaning in the sense of traces.

Let us first deal with problem (1).

Lemma 8. Let $\Omega$ and $\mathbf{f}$ be as in Section 2. Then the problem of finding $\mathbf{u} \in V$ satisfying (1) is equivalent to solving the weak problem (5).

Proof. Let $\mathbf{u} \in V$ be a solution of (1). Then $-\triangle \mathbf{u}=\operatorname{curl} \operatorname{curl} \mathbf{u} \in L^{2}(\Omega)$ ) and so the formal calculations leading to (5) can be rigorously justified.

Conversely, if $\mathbf{u} \in V$ satisfies (5), then by using $\mathbf{v} \in \mathcal{D}(\Omega)^{N}$ and transferring all derivatives to $\mathbf{v}$ we get $-\triangle \mathbf{u}=\mathbf{f}$ in the sense of distributions. For any $\phi \in \mathcal{D}(\Omega)$ we can find $g \in H_{0}^{1}(\Omega)$ such that $\triangle g=\phi$. Now use $\mathbf{v}=\nabla g$ in the weak form (note that $\nabla g \in V$ ). We get

$$
\int_{\Omega}(\operatorname{div} \mathbf{u}) \phi=\int_{\Omega} \mathbf{f} \cdot \nabla g=-\int_{\Omega}(\operatorname{div} \mathbf{f}) g+\int_{\Gamma}(\mathbf{f} \cdot \mathbf{n}) g=0,
$$

so $\operatorname{div} \mathbf{u}=0$ in the sense of distributions. Consequently the problem (1) (in $V$ ) and the weak form (5) are equivalent.

Corollary 1. The problem (1) has a unique solution $\mathbf{u}_{V}$ in $V$.

In the proof of the theorem we used the fact that $\nabla g \in V$. If we were dealing with the weak form in $H_{0 t}^{1}(\Omega)$, then $\nabla g$ may not have been a legitimate test function (in the case $g \notin H^{2}(\Omega)$ ), which would make it impossible to prove that $\operatorname{div} \mathbf{u}=0$. This is the difference between the weak problems (5) and (9), even though they appear very similar.

The corresponding proof for the problem (3) is a little more difficult, but follows the same pattern.

Lemma 9. Let $\Omega$ and $\mathbf{f}$ be as in Section 2. Assume in addition that the boundary $\Gamma$ is piecewise $C^{1,1}$ and the jump condition (6) is satisfied. Then the problem of finding $\mathbf{u} \in H_{0 t}^{1}(\Omega)$ satisfying (3) is equivalent to solving the weak problem (7).

Proof. We first have to show that the boundary condition

$$
\frac{\partial \mathbf{u}}{\partial \mathbf{n}} \cdot \mathbf{n}+\kappa \mathbf{u} \cdot \mathbf{n}=0 \text { on } \Gamma
$$


has a meaning for $\mathbf{u} \in H_{0 t}^{1}(\Omega)$ with $\triangle \mathbf{u} \in L^{2}(\Omega)$. Since $\nabla \mathbf{u} \in L^{2}(\Omega)^{N}$ and $\operatorname{div} \nabla \mathbf{u} \in L^{2}(\Omega)$, the gradient has a normal trace on the boundary $\frac{\partial \mathbf{u}}{\partial \mathbf{n}} \in H^{-1 / 2}(\Gamma)$ (see [2] for example). For $\phi \in H_{00}^{1 / 2}\left(\Gamma_{j}\right)$ we have $\phi \mathbf{n} \in H^{1 / 2}(\Gamma)$ (using the extension of $\phi$ by zero) and therefore the boundary condition may be interpreted as

$$
\left\langle\frac{\partial \mathbf{u}}{\partial \mathbf{n}}+\kappa \mathbf{u}, \phi \mathbf{n}\right\rangle_{\Gamma}=0 \quad \forall \phi \in \prod_{j=1}^{n} H_{00}^{1 / 2}\left(\Gamma_{j}\right),
$$

or, equivalently,

$$
\left\langle\frac{\partial \mathbf{u}}{\partial \mathbf{n}}+\kappa \mathbf{u}, \mathbf{v}\right\rangle_{\Gamma}=0 \quad \forall \mathbf{v} \in H_{0 t}^{1}(\Omega)
$$

where $\langle\cdot, \cdot\rangle_{\Gamma}$ is the duality pairing between $H^{-1 / 2}(\Gamma)$ and $H^{1 / 2}(\Gamma)$.

Let $\mathbf{u} \in H_{0 t}^{1}(\Omega)$ be a solution of (3). Using (12) we can justify to formal calculations leading to the weak form $(7)$ (the boundary integrals in the weak form should be understood as duality pairings between $\left(H_{00}^{1 / 2}\left(\Gamma_{j}\right)\right)^{\prime}$ and $\left.H_{00}^{1 / 2}\left(\Gamma_{j}\right)\right)$.

Conversely, if $\mathbf{u} \in H_{0 t}^{1}(\Omega)$ satisfies (7), then by using $\mathbf{v} \in \mathcal{D}(\Omega)^{N}$ we get as before $-\triangle \mathbf{u}=\mathbf{f}$ in the sense of distributions. For any $\mathbf{v} \in H_{0 t}^{1}(\Omega)$, integrating by parts in the weak form (note that $\triangle \mathbf{u} \in L^{2}(\Omega)^{N}$ ) we get

$$
-\int_{\Omega} \triangle \mathbf{u} \cdot \mathbf{v}+\left\langle\frac{\partial \mathbf{u}}{\partial \mathbf{n}}, \mathbf{v}\right\rangle_{\Gamma}+\sum_{i=1}^{n} \int_{\Gamma_{j}} \kappa \mathbf{u} \cdot \mathbf{v}=\int_{\Omega} \mathbf{f} \cdot \mathbf{v}
$$

hence (12) is satisfied. Consequently the problem (3) (in $H_{0 t}^{1}(\Omega)$ ) and the weak form (7) are equivalent.

Corollary 2. The problem (3) has a unique solution $\widetilde{\mathbf{u}}$ in $H_{0 t}^{1}(\Omega)$.

Now we will show that problems (2) and (3) are equivalent. The only difference in these two problems is in the boundary condition, so we have to show that the divergence boundary condition makes sense and is equivalent to the boundary condition in (3). This is done in the following lemma.

Lemma 10. Assume $\Omega$ satisfies the conditions of Lemma 9. For $\mathbf{u} \in H_{0 t}^{1}(\Omega)$ with $\triangle \mathbf{u} \in L^{2}(\Omega)^{N}$, and any $j \in\{1, \ldots, n\}$, the trace of $\operatorname{div} \mathbf{u}$ on $\Gamma_{j}$ exists and belongs to $\left(H_{00}^{1 / 2}\left(\Gamma_{j}\right)\right)^{\prime}$, and

$$
\left.\operatorname{div} \mathbf{u}\right|_{\Gamma_{j}}=\left.\frac{\partial \mathbf{u}}{\partial \mathbf{n}} \cdot \mathbf{n}\right|_{\Gamma_{j}}+\left.\kappa \mathbf{u} \cdot \mathbf{n}\right|_{\Gamma_{j}}
$$

Proof. This follows from Lemma (7) which gives the formula for smooth $\mathbf{u}$ without the condition $\mathbf{u} \times \mathbf{n}=0$. By density this can be extended to $\mathbf{u} \in H^{1}(\Omega)^{N}$ with $\triangle \mathbf{u} \in L^{2}(\Omega)^{N}$. Then use the fact that for $\mathbf{u} \in H_{0 t}^{1}(\Omega)$ we have $\operatorname{div}{ }_{\Gamma} \mathbf{u}_{\tau}=0$ (see the end of proof of Lemma 4 ).

Corollary 3. Problems (2) and (3) are equivalent.

Let us summarize the results in a theorem:

Theorem 2. Assume that $\Omega$ is a bounded subset of $\mathbf{R}^{N}$ with a connected, Lipschitz boundary. Let $\mathbf{f} \in L^{2}(\Omega)^{N}$ with $\operatorname{div} \mathbf{f}=0$ be given. Then

a) The problem (1) in $V$ is equivalent to the weak form (5) and has a unique solution $\mathbf{u}_{V} \in V$.

b) If in addition the boundary is piecewise $C^{1,1}$ and satisfies the jump condition (6), then the problems (2) and (3) in $H_{0 t}^{1}(\Omega)$ are both equivalent to weak problems (7) and (9), and have a unique solution $\mathbf{u}_{H} \in H_{0 t}^{1}(\Omega)$. 


\section{Relationship of the $\mathrm{V}$ and $\mathrm{H}_{0 t}^{1}(\Omega)$ FORmulations}

Let $\mathbf{u}_{V} \in V$ be the solution of (5) (or the strong form (1 with the interior divergence condition) and let $\mathbf{u}_{H} \in H_{0 t}^{1}(\Omega)$ be the solution of (9) (which under the additional smoothness conditions is equivalent to problems (2) and (3) with the divergence boundary condition). In this section we will discuss when the two solutions are the same. The following is clearly true.

Lemma 11. Let $\Omega$ and $\mathbf{f}$ be as in Section 2. Assume in addition that the jump condition (6) is satisfied. Then the following are equivalent

a) $\mathbf{u}_{V}=\mathbf{u}_{H}$;

b) $\operatorname{div} \mathbf{u}_{H}=0$ in $\Omega$;

c) $\mathbf{u}_{V} \in H_{0 t}^{1}(\Omega)$.

Let us now examine when $\mathbf{u}_{V}=\mathbf{u}_{H}$. First, note that Lemma 5 implies that $H_{0 t}^{1}(\Omega)$ is closed in $V$. We have two possibilities: either $V=H_{0 t}^{1}(\Omega)$ or $V \neq H_{0 t}^{1}(\Omega)$. In the first case the solutions are obviously the same (the corresponding weak forms are exactly the same). The following lemma gives the necessary and sufficient conditions for the equality of the spaces.

Lemma 12. Assume that $\Omega$ is a bounded subset of $\mathbf{R}^{N}$ with a connected Lipschitz boundary. The spaces $V$ and $H_{0 t}^{1}(\Omega)$ are equal if and only if the homogeneous Dirichlet problem for the scalar Poisson equation with any right hand side in $\mathcal{D}(\Omega)$ has a solution in $H^{2}(\Omega)$.

Proof. Suppose that the regularity condition holds. For any given $\mathbf{w} \in V$ we can solve the weak problem: find $\mathbf{u} \in H_{0 t}^{1}(\Omega)$ such that

$$
a(\mathbf{u}, \mathbf{v})=a(\mathbf{w}, \mathbf{v}) \quad \forall \mathbf{v} \in H_{0 t}^{1}(\Omega)
$$

(i.e. $\mathbf{u}$ is a projection of $\mathbf{w}$ onto $H_{0 t}^{1}(\Omega)$ corresponding to the scalar product $a(\cdot, \cdot)$ ). As in the proof of Lemma 8 we can show that $\triangle(\mathbf{u}-\mathbf{w})=0$, and using the $H^{2}$-regularity of the solution of the Dirichlet problem for the Poisson equation we also get $\operatorname{div}(\mathbf{u}-\mathbf{w})=0$. Since $(\mathbf{u}-\mathbf{w}) \times\left.\mathbf{n}\right|_{\Gamma}=0$, by the uniqueness of solution of (1) we have $\mathbf{u}=\mathbf{w}$, i.e. $\mathbf{w} \in H_{0 t}^{1}(\Omega)$. Consequently $V=H_{0 t}^{1}(\Omega)$.

Now suppose that there is $g \in H_{0}^{1}(\Omega)$ such that $\triangle g \in \mathcal{D}(\Omega)$, but $g \notin H^{2}(\Omega)$. Then $\nabla g \in V$, but $\nabla g \notin H_{0 t}^{1}(\Omega)$, so $V \neq H_{0 t}^{1}(\Omega)$.

Note that the lemma remains true if one substitutes $L^{2}(\Omega)$ for $\mathcal{D}(\Omega)$.

Corollary 4. If $\Omega$ is convex or has a $C^{1,1}$ boundary then $V=H_{0 t}^{1}(\Omega)$.

This follows from the classical regularity theory about the smoothness of the solution of the Dirichlet problem for the Poisson equation. It is actually enough to assume that $\Omega$ is locally convex near the points where the boundary is not $C^{1,1}$ (i.e. these points have a neighborhood whose intersection with $\Omega$ is convex).

The question of the equality of these spaces (and analogous ones with a boundary condition for the normal trace) has been partly answered by different authors, but we are unaware of results as sharp as these presented here. In the classical book [2] it is proved that $V=H_{0 t}^{1}(\Omega)$ if $\Omega$ is a convex polygon or has a $C^{1,1}$ boundary. Necessary and sufficient conditions for $V=H_{0 t}^{1}(\Omega)$ to hold in $\mathbf{R}^{2}$ are given in [6], namely that the scalar Poisson equation with $L^{2}(\Omega)$ right hand side must have a solution in $H^{2}(\Omega)$ both with Dirichlet and Neumann condition. As we saw, the regularity of solution of the Neumann problem is not needed, and in $\mathbf{R}^{2}$ it actually follows from the regularity of solution of the Dirichlet problem.

In the other case, when $H_{0 t}^{1}(\Omega)$ and $V$ are not identical, since $H_{0 t}^{1}(\Omega)$ is closed in $V$, there is $\mathbf{f} \in L^{2}(\Omega)$ with $\operatorname{div} \mathbf{f}=0$ for which the solution of $(1)$ is in $V$, but not in $H_{0 t}^{1}(\Omega)$. This means that the interior and boundary divergence formulations are not equivalent and give different solutions for this $\mathbf{f}$. We will show how to construct f for which $\mathbf{u}_{V} \neq \mathbf{u}_{H}$ whenever $V \neq H_{0 t}^{1}(\Omega)$.

Lemma 13. If $V \neq H_{0 t}^{1}(\Omega)$ than there is $\mathbf{f} \in L^{2}(\Omega)$ with $\operatorname{div} \mathbf{f}=0$ such that the corresponding solution of (1) is not in $H_{0 t}^{1}(\Omega)$. 
Proof. If $V \neq H_{0 t}^{1}(\Omega)$ then we can choose a nonzero $\mathbf{w} \in V$ such that

$$
a(\mathbf{w}, \mathbf{v})=0 \quad \forall \mathbf{v} \in H_{0 t}^{1}(\Omega)
$$

First note that curl $\mathbf{w} \neq 0$ : indeed, if we had $\operatorname{curl} \mathbf{w}=0$ then

$$
\int_{\Omega} \operatorname{div} \mathbf{w} \cdot \operatorname{div} \mathbf{v}=0 \quad \forall \mathbf{v} \in H_{0 t}^{1}(\Omega)
$$

and hence (by using $\left.\mathbf{v} \in \mathcal{D}(\Omega)^{N}\right)$ ), $\nabla \operatorname{div} \mathbf{w}=0$, or $\operatorname{div} \mathbf{w}=$ const. It follows from (15) that $\int_{\Omega} \operatorname{div} \mathbf{v}=0$ for all $\mathbf{v} \in H_{0 t}^{1}(\Omega)$, which is a contradiction.

Now let $\mathbf{f} \in V$ be a solution of

$$
a(\mathbf{f}, \mathbf{v})=\int_{\Omega} \operatorname{curl} \mathbf{w} \cdot \operatorname{curl} \mathbf{v} \forall \mathbf{v} \in V .
$$

Note that $\mathbf{f} \neq 0$ and $\operatorname{div} \mathbf{f}=0$. We claim that for this $\mathbf{f}$ problem (1) has a solution $\mathbf{u}_{V} \in V$ which is not in $H_{0 t}^{1}(\Omega)$. Indeed, using the weak problem (5) and (16) we get

$$
a(\mathbf{u}, \mathbf{w})=\int_{\Omega} \operatorname{curl} \mathbf{u} \cdot \operatorname{curl} \mathbf{w}=a(\mathbf{u}, \mathbf{f})=\int_{\Omega}|\mathbf{f}|^{2} \neq 0,
$$

which by (14) implies that $\mathbf{u}_{V} \notin H_{0 t}^{1}(\Omega)$.

We can also give a more tangible example of a solution of (1) which does not belong to $H_{0 t}^{1}(\Omega)$. Suppose $\Omega \subset \mathbf{R}^{3}$ is such that in a neighborhood of some $\mathbf{x}_{0} \in \Gamma$ its boundary consists of 2 planes with the interior angle $\alpha>\pi$. Choose a cylindrical coordinate system $(r, \theta, z)$ with the origin at $\mathbf{x}_{0}, z$-axis along the edge and $\theta=0$, $\theta=\alpha$ on the boundary. Let $\phi \in C^{\infty}(\bar{\Omega})$, depending on $r$ and $z$ only, be such that $\phi \equiv 1$ near the origin and $\phi \equiv 0$ outside a neighborhood of the origin where the pieces of $\Gamma$ are planar. Put

$$
\mathbf{u}(x, y, z)=\operatorname{curl}\left(\phi(r, z) r^{\pi / \alpha} \cos \frac{\pi \theta}{\alpha} \mathbf{e}_{z}\right)
$$

where $\mathbf{e}_{z}$ is the unit vector in direction of $z$-axis. Then $\operatorname{div} \mathbf{u}=0$ and

$$
\mathbf{u} \times \mathbf{n}=\frac{\partial}{\partial \mathbf{n}}\left(\phi(r, z) r^{\pi / \alpha} \cos \frac{\pi \theta}{\alpha}\right) \mathbf{e}_{z}=0 \text { on } \Gamma .
$$

It follows that $\mathbf{u}$ is a solution of (1) with $\mathbf{f}=-\triangle \mathbf{u} \in L^{2}(\Omega)$ and $\operatorname{div} \mathbf{f}=0$. In addition, $\mathbf{f} \equiv 0$ near the origin. Since $\mathbf{u} \notin H_{0 t}^{1}(\Omega)$ and the problem (2) with the same $\mathbf{f}$ has a solution $\widetilde{\mathbf{u}} \in H_{0 t}^{1}(\Omega)$, we must have $\widetilde{\mathbf{u}} \neq \mathbf{u}$ and $\operatorname{div} \widetilde{\mathbf{u}} \neq 0$.

This example is essentially two-dimensional. To make it work for $\Omega \subset \mathbf{R}^{2}$ in a neighborhood of a corner with the interior angle $\alpha>\pi$ we just use $\phi$ independent of $z$. Note that the third component of $\mathbf{u}$ is zero, and we get $\mathbf{f} \in C^{\infty}(\bar{\Omega})^{2}$.

We will state these results as a theorem:

Theorem 3. Assume that $\Omega$ is a bounded subset of $\mathbf{R}^{N}$ with a Lipschitz boundary. The following are equivalent:

a) $\mathbf{u}_{V}=\mathbf{u}_{H}$ for any $\mathbf{f} \in L^{2}(\Omega)^{N}$ with $\operatorname{div} \mathbf{f}=0$;

b) $V=H_{0 t}^{1}(\Omega)$;

c) the Dirichlet problem for the scalar Poisson equation with any smooth right hand side has a solution in $H^{2}(\Omega)$. 


\section{Generalization to Helmholtz equation}

Most of the arguments above remain true in case of the vector Helmholtz equation. The only difference is in the coercivity, which now will not be sufficient for using Lax-Milgram. Let us sketch the results briefly. The analog of problem (1) is now: find $\mathbf{u} \in V$ such that

$$
\begin{cases}-\triangle \mathbf{u}-k^{2} \mathbf{u}=\mathbf{f} & \text { in } \Omega, \\ \operatorname{div} \mathbf{u}=0 & \text { in } \Omega, \\ \mathbf{u} \times \mathbf{n}=0 & \text { on } \Gamma .\end{cases}
$$

We assume that $\mathbf{f} \in L^{2}(\Omega)^{N}$ and the compatibility condition div $\mathbf{f}=0$ is satisfied. To formulate the weak problem let us define

$$
b(\mathbf{u}, \mathbf{v})=\int_{\Omega} \operatorname{curl} \mathbf{u} \cdot \operatorname{curl} \mathbf{v}+\operatorname{div} \mathbf{u} \operatorname{div} \mathbf{v}-k^{2} \mathbf{u} \cdot \mathbf{v} \quad \forall \mathbf{u}, \mathbf{v} \in V .
$$

Then the weak problem corresponding to (17) is to find $\mathbf{u} \in V$ such that

$$
b(\mathbf{u}, \mathbf{v})=\int_{\Omega} \mathbf{f} \cdot \mathbf{v} \forall \mathbf{v} \in V .
$$

The coercivity result is that $b(\mathbf{u}, \mathbf{u}) \geq C\|\mathbf{u}\|_{V}^{2}-k^{2}\|\mathbf{u}\|_{L^{2}}^{2}$ (if $k^{2}$ is complex, we take the real part). We cannot use Lax-Milgram directly. Instead we can use the Fredholm alternative to show the existence and uniqueness of the solution. Define an operator $A_{V}: L^{2}(\Omega) \rightarrow L^{2}(\Omega)$ by the requirement that $A_{V} \mathbf{g} \in V$ is the solution of $a(\mathbf{u}, \mathbf{v})=\int_{\Omega} \mathbf{g} \cdot \mathbf{v} \forall \mathbf{v} \in V$ (note that we do not need the compatibility condition $\operatorname{div} \mathbf{g}=0$ to guarantee the solvability of the weak problem). By the Lax-Milgram Lemma and the compact embedding theorem (Theorem 1) $A_{V}$ is a compact operator. Then the solution of (18) is the solution of $\mathbf{u}=A_{V}\left(k^{2} \mathbf{u}+\mathbf{f}\right)$, and if $1 / k^{2}$ is not an eigenvalue of $A_{V}$ then (18) has a unique solution $\mathbf{u} \in V$. The operator $A_{V}$ has two kinds of eigenfunctions: those that are divergence-free, and others which are gradients of the eigenfunctions of the scalar Laplacian with Dirichlet boundary conditions. Assuming that $1 / k^{2}$ is not an eigenvalue of $A_{V}$, one can prove the equivalence of the strong and weak problems similarly to the proof of Lemma 8 (in proving that the solution of the weak form satisfies the divergence condition we need solvability of the scalar Helmholtz equation with Dirichlet boundary conditions, which follows from the fact that if $1 / k^{2}$ is not an eigenvalue of $A_{V}$, then it is not an eigenvalue of the scalar Laplacian with Dirichlet boundary conditions).

The analog of problem (2) is to find $\mathbf{u} \in H_{0 t}^{1}(\Omega)$ such that

$$
\begin{cases}-\Delta \mathbf{u}-k^{2} \mathbf{u}=\mathbf{f} & \text { in } \Omega \\ \operatorname{div} \mathbf{u}=0 & \text { on } \Gamma \\ \mathbf{u} \times \mathbf{n}=0 & \text { on } \Gamma\end{cases}
$$

and the corresponding weak problem is to find $\mathbf{u} \in H_{0 t}^{1}(\Omega)$ such that

$$
b(\mathbf{u}, \mathbf{v})=\int_{\Omega} \mathbf{f} \cdot \mathbf{v} \quad \forall \mathbf{v} \in H_{0 t}^{1}(\Omega) .
$$

One can deal with the coercivity as above, using the operator $A_{H}: L^{2}(\Omega) \rightarrow L^{2}(\Omega)$ defined by: $A_{H} \mathbf{g} \in H_{0 t}^{1}(\Omega)$ is the solution of $a(\mathbf{u}, \mathbf{v})=\int \mathbf{g} \cdot \mathbf{v} \forall \mathbf{v} \in H_{0 t}^{1}(\Omega)$. Equivalence of the strong and weak forms follows similarly to the zero frequency case.

The question of when the two weak problems (18) and (20) give the same solution can be answered as in Section 7. Most of the results presented there do not depend on the frequency. The counterexamples are also 
easy to modify: if $\mathbf{u}_{V} \notin H_{0 t}^{1}(\Omega)$ is a solution of (1), then it is also a solution of (17) with the right hand side $\mathbf{f}-k^{2} \mathbf{u}_{V}$. Theorem 3 remains true if we assume in addition that $1 / k^{2}$ is not an eigenvalue of either $A_{V}$ or $A_{H}$ and let $\mathbf{u}_{V}$ and $\mathbf{u}_{H}$ be the solutions of the weak problems (18) and (20) correspondingly.

Similar results can be proved for time-dependent problems, such as the vector wave equation and Maxwell's equations. Even with divergence-free initial conditions, using a weak form in $H_{0 t}^{1}(\Omega)$ (similar to (20)) may give a solution not satisfying the divergence constraint. Again, this can happen only if the $H^{2}$-regularity of the Dirichlet problem for the scalar Poisson equation fails.

\section{Conclusions}

We saw that in some cases (e.g. for nonconvex polygons) the boundary divergence formulation is not equivalent to the interior divergence formulation, and the weak form (9) or (20) (the weak forms in $H_{0 t}^{1}(\Omega)$ ), while uniquely solvable, may not have a divergence-free solution. In this case approximation methods based on these weak formulations (e.g. the usual finite element method, the least squares method) will converge to a spurious solution (not satisfying the divergence constraint). To avoid the spurious solution, one should use the weak form in $V$, but this is not straightforward: one has to use basis functions which are in $V$, but not in $H_{0 t}^{1}(\Omega)$ (e.g. incorporating singular solutions around the re-entrant corners), in addition to the usual ones. The same is true of the penalty method, where the weak form contains a penalty parameter in front of the divergence term. If the solution of the original problem is not in $H_{0 t}^{1}(\Omega)$, then any choice of the penalty parameter will result in a spurious solution - in fact, the smaller one makes the divergence (by choosing larger values of the parameter), the bigger will be the error in the curl of the solution.

On the other hand, when the formulations are equivalent (this is the case when the solution of the Dirichlet problem for the scalar Poisson equation is in $H^{2}(\Omega)$ ), one can use weak forms in $H_{0 t}^{1}(\Omega)$. This permits the use of simpler algorithms, e.g. finite element methods with piecewise linear test and trial functions. The equivalent formulation in terms of gradients (as in (7)) may be especially useful, since it decouples the field components inside the domain.

\section{REFERENCES}

[1] M. Costabel, A Remark on the Regularity of Solutions of Maxwell's Equations on Lipschitz Domains. Math. Methods Appl. Sci. 12 (1990) 365-368.

[2] V. Girault and P.-A. Raviart, Finite Element Methods for Navier-Stokes Equations. Springer-Verlag (1986).

[3] P. Grisvard, Elliptic Problems in Nonsmooth Domains. Pitman Advanced Publishing Program (1985).

[4] C. Hazard and M. Lenoir, On the Solution of Time-harmonic Scattering Problems for Maxwell's Equations. SIAM J. Math. Anal. 27 (1996) 1597-1630.

[5] B.N. Jiang, J. Wu and L.A. Povinelli, The Origin of Spurious Solutions in Computational Electromagnetics. J. Comput. Phys. 125 (1995) 104-123.

[6] M. Křížek and P. Neittaanmäki, On the Validity of Friedrichs' Inequalities. Math. Scand. 54 (1984) 17-26.

[7] I.D. Mayergoyz, A New Point of View on the Mathematical Structure of Maxwell's Equations. IEEE Trans. Magn. 29 (1993) 1315-1320.

[8] F. Murat, Compacité par Compensation. Ann. Scuola Norm. Sup. Pisa Cl. Sci. 5 (1978) 485-507. 